\title{
The Effect of YiQiFuMai on Ischemic Heart Failure by Improve Myocardial Microcirculation and Increase eNOS and VEGF Expression
}

\author{
Shuren Li*, Xiao Hao, Sa Xiao, Liying Xun \\ Department of Heart Center, Hebei General Hospital, Shijiazhuang, China \\ Email: *1sr64@126.com
}

How to cite this paper: Li, S.R., Hao, X., Xiao, S. and Xun, L.Y. (2020) The Effect of YiQiFuMai on Ischemic Heart Failure by Improve Myocardial Microcirculation and Increase eNOS and VEGF Expression. International Journal of Clinical Medicine, 11, 84-100.

https://doi.org/10.4236/ijcm.2020.112009

Received: January 14, 2020

Accepted: February 17, 2020

Published: February 20, 2020

Copyright $\odot 2020$ by author(s) and Scientific Research Publishing Inc. This work is licensed under the Creative Commons Attribution International License (CC BY 4.0).

http://creativecommons.org/licenses/by/4.0/

(c) (i) Open Access

\begin{abstract}
Objective: To assess the effects of traditional Chinese medicine YiQiFuMai on cardiac function during the progression of ischemic heart failure. Methods: Rabbits were divided into sham, heart failure, and YiQiFuMai groups. The ischemic heart failure model was established in New Zealand white rabbits, which were intraperitoneally injected with YiQiFuMai injection and $0.9 \%$ sodium chloride after the operation. After six weeks, cardiac function was examined by ultrasound; serum BNP levels were measured by ELISA; p-AKT, eNOS, ICAM-1 and VEGF levels were evaluated by real-time PCR and Western-Blot; pathological changes of the myocardial tissue were observed by H\&E staining; CD31 expression in tissue samples was analyzed by immunohistochemistry. The ultrastructure and microcirculation of myocardial tissue specimens from the three groups were assessed by transmission electron microscopy. Results: YiQiFuMai decreased serum BNP levels, and increased LVEF and reduced LVEDD at 6 weeks postoperatively. In addition, YiQiFuMai can improve myocardial damage and microcirculation structure, as assessed by histology and transmission electron microscope. At the molecular level, treatment with YiQiFuMai resulted in increased eNOS, VEGF and p-AKT levels but reduced ICAM-1 amounts compared with the heart failure group. Conclusion: Ischemic heart failure damages the microvascular structure and functions of the myocardium. Treatment with YiQiFuMai potentially ameliorates microcirculatory damage and alleviates cardiac failure by improving endothelial function and angiogenesis, and inhibiting inflammatory cell adhesion.
\end{abstract}

\section{Keywords}

Endothelial Nitric Oxide Synthase (eNOS), Endothelial Cells, PI3K/AKT/eNOS Pathway, Microcirculation, Heart Failure, YiQiFuMai 


\section{Introduction}

After acute myocardial infarction, myocardial fibrosis and cardiac dilatation occur gradually in the infarcted myocardium, eventually leading to heart failure [1]. Coronary ischemia causes hemodynamic disorder, excessive neuroendocrine activation and abnormalities in energy metabolism, which aggravate ventricular remodeling and cardiac dysfunction [2].

It has been highlighted that microcirculatory dysfunction plays a pivotal role in ischemic heart failure [3]. eNOS is required for producing NO, an important vasodilator substance that is indispensable to normal cardiac function. Its release is affected by a series of signaling pathways during endothelial injury, leading to coronary microvascular damage [4]. Endothelial damage is associated with excessive oxidative stress and inflammatory response, as well as upregulation of adhesion molecules such as ICAM-1 and VCAM-1, resulting in platelet aggregation and adhesion that affect microcirculation [5].

Microvascular angiogenesis is crucial to survival and functional recovery after ischemic heart disease. Angiogenesis is triggered by angiogenic factors binding to endothelial cell receptors, and VEGF is the major factor contributing to angiogenesis [6]. VEGF plays an important role in angiogenesis through three structurally related VEGF receptor tyrosine kinases, including VEGFR1, VEGFR2 and VEGFR3 [7]. VEGFR2, a major VEFG receptor in endothelial cells, is indispensable for the differentiation, proliferation and migration of endothelial cells, as well as angiogenesis. Additionally, VEGF can increase vascular permeability to facilitate angiogenesis by binding to VEGFR2. VEGF is critically involved in the maturation of newly formed blood vessels in ischemic myocardium, providing long-term blood supply for cardiomyocytes and limiting ventricular remodeling after myocardial infarction [8].

A variety of drugs are currently used to improve cardiac microcirculation. Yiqifumai Injection (YQFM), is composed of Panax ginseng, Ophiopogon japonicas, and Schisandra chinensis (Ginseng). Li, F., et al. found schisandrin pretreatment inhibited cell apoptosis, as evidenced by inhibiting activation of caspase-3 and increasing the Bcl-2/Bax ratio. Meanwhile, the vascular endothelium protective property of schisandrin might be beneficial for the treatment of cardiovascular disease [9]. A study by China demonstrated that YQFM could ameliorate myocardial apoptosis via positive regulation of AMPK, PI3 K/Akt and negative regulation of MAPKs signaling pathways. Besides, ginsenoside Rd might partially adjust omentin-dependent protective effect of YQFM [10]. In addition, they also found that YQFM markedly attenuated mitochondrial dysfunction through improving mitochondrial morphology, increasing mitochondria membrane potential $(1 \mu \mathrm{m})$, mitochondrial ROS generation and expression of Mitofusin-2 (Mfn2), meanwhile, decreasing phosphorylation of dynamin-related protein 1 (p-Drp1) [11]. In this study, we assessed the beneficial effects of YiQiFuMai on myocardial microcirculation in rabbits during ischemic heart failure. We showed that treatment with YiQiFuMai resulted in increased levels of the protective molecules eNOS and VEGF, while downregulating ICAM-1. 


\section{Materials and Methods}

The schedule for animal experiments is as shown in Figure 1.

\section{Experimental animals}

A total of 30 healthy New Zealand male rabbits aged 3 months (approximately $2.5 \pm 0.3 \mathrm{~kg}$ ) were used in this study. The animals were provided by the Experimental Animal Center of Hebei Medical University, and randomly divided into the sham operation, heart failure, YiQiFuMai groups, with 10 animals per group. After successful establishment of the heart failure model, YiQiFuMai injection (lyophilized) (Tianjin Zhijiao Pharmaceutical Co., Ltd. of Tasly Holding Group) at a concentration of $270 \mathrm{mg} / \mathrm{mL}$ in normal saline was intraperitoneally administered at $2 \mathrm{~mL} /(\mathrm{kg} \cdot \mathrm{d})$ for 4 weeks in the YiQiFuMai group. The sham operation and heart failure groups were intraperitoneally administered normal saline at $2 \mathrm{~mL} /(\mathrm{kg} \cdot \mathrm{d})$ for 4 weeks.

\section{Establishment of the experimental animal model}

The ischemic heart failure model was established by ligating the anterior descending coronary artery of rabbits [12]. After weighing, sodium pentobarbital anesthesia was performed via the ear vein. Preoperative ECG was recorded using an electrocardiograph. Skin and subcutaneous tissues were cut along the left margin of the sternum. The pericardium was cut off to expose the anterior descending artery of the heart. The anterior descending branch was ligated using No.0 lines, 2 - $3 \mathrm{~mm}$ below the junction between the pulmonary conus and the left atrial appendage. Then, the chest was sutured. In the sham operation group, only the chest was open, and the anterior descending coronary artery was not ligated. After modeling, the apex and the left ventricular anterior wall did not change in color, myocardial motion did not decrease, and precordial ST segment did not raise. After the operation, all rabbits were intramuscularly injected 800,000 units of penicillin daily for a total of 3 days. Cardiac ultrasonography was performed 2 weeks after modeling, and the ischemic heart failure model was considered to be successfully established with LVEF below $50 \%$.

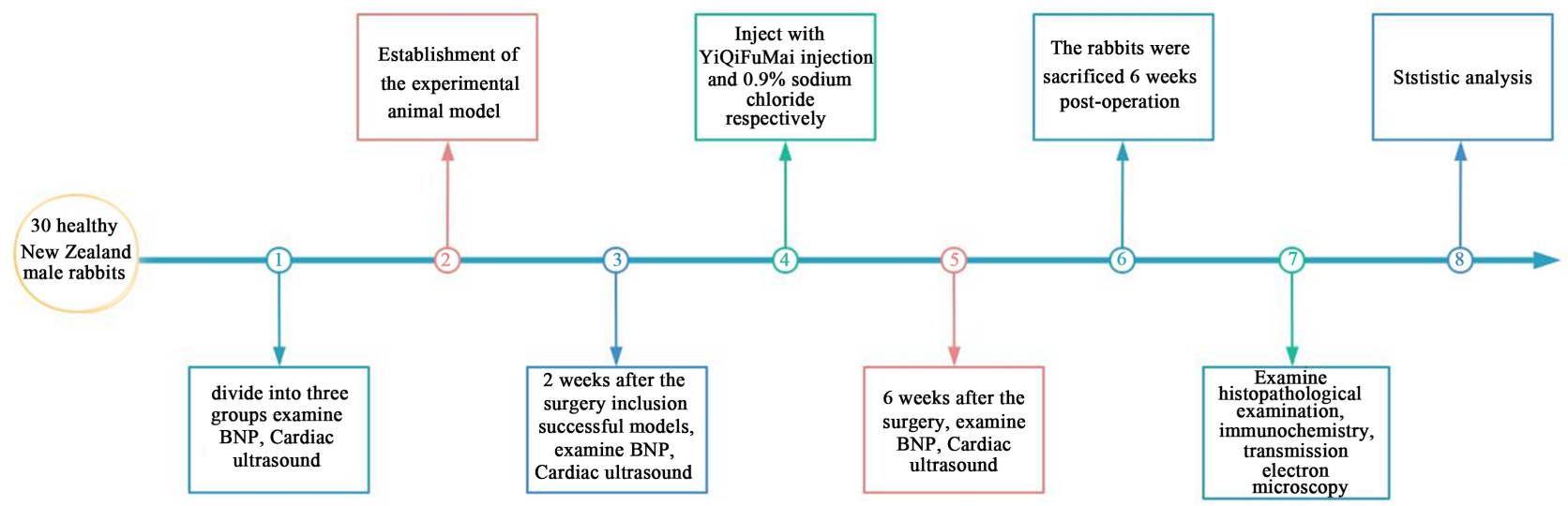

Figure 1. The schedule for animal experiments. 


\section{Cardiac function test by cardiac ultrasonography}

Cardiac function was tested by the same cardiac sonographer using a cardiac echocardiography device (ALOKA) 2 weeks before the operation and 6 weeks postoperatively. The rabbits were fixed on a table to remove chest fur to expose the skin. The ultrasonic probe was replaced with a cardiac ultrasound probe at a frequency of 5.0 MHZ. Ventricular wall motion was observed from the long-axis view of the sternum left ventricle. The mitral septal angle horizontally and the curve of left ventricular posterior wall were assessed by M-mode ultrasound to measure LVEF and LVEDD. Mean values from three measurements were calculated.

\section{Detection of serum BNP levels by ELISA}

A total of $4 \mathrm{ml}$ of blood was collected via the middle auricular artery from each animal before the operation, and 2 and 6 weeks post-operation, respectively, without anti-coagulation. Blood samples were placed at room temperature for $2 \mathrm{~h}$, then centrifuged at $3000 \mathrm{rpm}$ for $15 \mathrm{~min}$ for serum preparation. Operations were based on the ELISA kit (Shanghai Enzyme Biotechnology Co., Ltd.) instructions. A regression formula was generated according to the concentrations of standards and the corresponding absorbance values. BNP levels were derived based on the above formula.

\section{Histopathological examination}

Rabbits were sacrificed 6 weeks after the operation to harvest myocardial tissues in the infarcted border areas. Gradient dehydration was performed with ethanol after tissue fixation with paraformaldehyde. Dehydrated tissue samples were paraffin embedded and sliced transversely into $5 \mu \mathrm{m}$ sections using a slicer. Before staining, the tissue sections were dewaxed with xylene. Staining with $\mathrm{H} \&$ E followed routine procedures. Finally, observation was performed on an AHB-2-HL optical microscope (Olympus, Japan).

\section{Immunochemistry}

After slicing the paraffin blocks as described above, the sections were dewaxed and hydrated. Then, antigen retrieval, incubation with $3 \% \mathrm{H}_{2} \mathrm{O}_{2}$ in deionized water, and sealing with goat serum were performed, followed by incubation with mouse anti-rabbit CD31 primary antibodies (1:50) (BIOSS/bs-0195R) at $4^{\circ} \mathrm{C}$ overnight. Next, goat anti-mouse IgG II antibodies (Santa/Sc-2005) and streptavidin-horseradish peroxidase (S-A/HRP) were added dropwise for incubation at room temperature. Finally, DAB staining, conventional counterstaining, dehydration and film sealing with neutral gum were performed. The cytoplasm of positive vascular endothelial cells was stained brown.

\section{Assessment of myocardial tissue ultrastructure by transmission electron microscopy}

Six weeks post-operation, the whole heart was extracted by opening the chest once again, and rinsed thoroughly with cooled saline. Myocardial tissues of the left ventricular anterior wall were harvested along the direction of myocardial fibers in the infarcted border areas at a size of $1 \times 1 \times 3 \mathrm{~mm}^{3}$. The harvested myocardial tissue samples were immediately placed in $4 \%$ glutaraldehyde for 
overnight fixation, followed by rinsing, fixation, dehydration with gradient acetone, immersion, embedding and ultra-thin slicing after polymerization $(50 \mathrm{~nm})$. Tissue slices were successively stained with uranyl acetate for 30-45 min and lead citrate for 5 - 30 min. Observation was performed under a Hitachi H-7500 transmission electron microscope (Japan Hitachi, Ltd.).

\section{Real-time $q P C R$}

The rabbits were sacrificed 6 weeks post-operation. The myocardial tissue (50 $\mathrm{mg}$ ) from the infarcted area was homogenized for total RNA extraction. Reverse transcription was performed according to the reverse transcription kit's instructions. Primer sequences are shown in Table 1 . The obtained cDNA was subjected to real-time fluorescent quantitative PCR. $\beta$-actin was used as an internal reference, and the $2^{-\Delta \Delta \mathrm{Ct}}$ method was employed for analysis $(\Delta \mathrm{Ct}=\mathrm{Ct}$ value of the target gene-Ct value of $\beta$-actin; $\Delta \Delta \mathrm{Ct}=\Delta \mathrm{Ct}$ value of the target gene $-\Delta \mathrm{Ct}$ value of $\beta$-actin).

\section{Immunoblotting}

The rabbits were sacrificed 6 weeks post-operation. After weighing, myocardial tissue samples from the infarcted border areas were homogenized for protein extraction. Total protein was extracted with RIPA buffer supplemented with protease and phosphatase inhibitors. The samples were then subjected to polyacrylamide gel electrophoresis and transferred onto PVDF membranes, which were immersed in Western blot blocking solution. After incubation with primary antibodies (1:1000) raised against p-Akt (BIOSS/bs-0876R), eNOS (Abcam/ab5589), ICAM-1 (BIOSS/bs-4617R), VEGF (Abcam/ab1316) and $\beta$-actin (Abcam ab8226) at $4^{\circ} \mathrm{C}$ overnight, respectively, the membranes were washed and subjected to incubation with secondary antibodies (1:5000). ECL solution was used for development. The protein levels of eNOS, ICAM-1, p-AKT and VEGF were then quantified.

\section{Statistical analysis}

Data were analyzed with the SPSS 21.0 software. Continuous data with normal distribution were represented by mean \pm standard deviation (Mean \pm SD) and compared by one-way ANOVA. Pairwise comparisons of multiple samples were performed by the SNK-q test. $P<0.05$ was considered statistically significant.

Table 1. Primers for real-time PCR.

\begin{tabular}{cc}
\hline Primer & Sequences $\left(5^{\prime}-3^{\prime}\right.$ ) \\
\hline eNOS-Forward & ACCTTCGTTCAGCCATCACAGT \\
eNOS-Reverse & GACACCTCCAGGACCAACTCAG \\
ICAM-1-Forward & GACTGCCTGGGAAACTGGACTT \\
ICAM-1-Reverse & GCCGCCACCACGATGATGAT \\
VEGF-Forward & TTCATGGAAGTCTACCGGCG \\
VEGF-Reverse & TGACGTTGAACTCCTCGGTG \\
$\beta$-action-Forward & AGATCGTGCGGGACATCAAG \\
$\beta$-action-Reverse & CAGGAAGGAGGGCTGGAAGA
\end{tabular}




\section{Results}

\section{YiQiFuMai improve heart failure in the rabbit model}

A total of 30 New Zealand white rabbits were subjected to modeling, and 24 survived. In each group, 8 rabbits survived and 2 died. A total of 6 rabbits died. Among them, 2 animals died of pneumothorax, 2 of ventricular fibrillation, 1 of excessive anesthesia, and 1 of excessive bleeding due to puncture of the heart.

At 6 weeks after operation, the heart failure, YiQiFuMai groups showed markedly increased LVEF in comparison with the values at 2 postoperative weeks ( $\mathrm{P}$ $<0.05)$, while there were no significant differences in the sham group for LVEF and LVEDD at these times (Table 1). There were no significant differences for LVEDD in the heart failure group at these time points. LVEDD was significantly decreased in the YiQiFuMai groups. Compared with the heart failure group, the YiQiFuMai showed significantly increased LVEF, and markedly reduced LVEDD (Table 1).

There were no statistically significant differences in LVEF and LVEDD among groups before operation $(\mathrm{P}>0.05)$. Two weeks after the surgical operation, we found that LVEF values in the heart failure, YiQiFuMai groups were significantly increased compared with preoperative values, while LVEDD values in these three groups were significantly decreased after modeling (Table 2). Besides, LVEF and LVEDD significantly differed, with increased LVEF and reduced LVEDD in animals with heart failure (heart failure, YiQiFuMai groups) compared with the sham group (Table 2).

We analyzed the LVEDD and LVEF of each animal before operation, 2 weeks after operation, and 6 weeks after operation, as shown in the box chart (Figure 2).

\section{Serum BNP concentrations are reduced in the YiQiFuMai group}

Next, serum levels of BNP were assessed by ELISA. We found that there were no statistically significant differences among groups before operation. Two weeks after the operation, BNP concentrations in the sham operation group were not significantly different from those before modeling, while the heart failure, YiQiFuMai groups showed significantly increased serum BNP amounts compared with preoperative values. Compared with the sham operation group, the heart failure, YiQiFuMai groups showed significantly increased serum BNP levels at 2 weeks postoperatively (Figure 3 ).

Table 2. LVEF and LVEDD values preoperatively, and at 2 and 6 weeks after operation.

\begin{tabular}{ccccc}
\hline & Group & Sham Group & Heart failure group & YiQiFuMai group \\
\hline LVEF & Preoperative & $69.2 \pm 5.8$ & $65.3 \pm 4.1$ & $66.0 \pm 3.9$ \\
$(\%)$ & 2 weeks & $66.1 \pm 7.6^{*}$ & $43.7 \pm 5.1$ & $41.0 \pm 6.3$ \\
& 6 weeks & $67.3 \pm 7.0^{*}$ & $52.4 \pm 7.4$ & $59.7 \pm 7.4^{*}$ \\
LVEDD & Preoperative & $12.5 \pm 1.4$ & $12.5 \pm 1.7$ & $12.5 \pm 1.6$ \\
$(\mathrm{~mm})$ & 2 weeks & $12.3 \pm 1.1^{*}$ & $14.0 \pm 1.2$ & $13.5 \pm 0.8$ \\
& 6 weeks & $11.3 \pm 1.9^{*}$ & $13.5 \pm 2.2$ & $11.7 \pm 1.3^{\star}$ \\
\hline
\end{tabular}

${ }^{\star} \mathrm{P}<0.05$ vs heart failure group. 


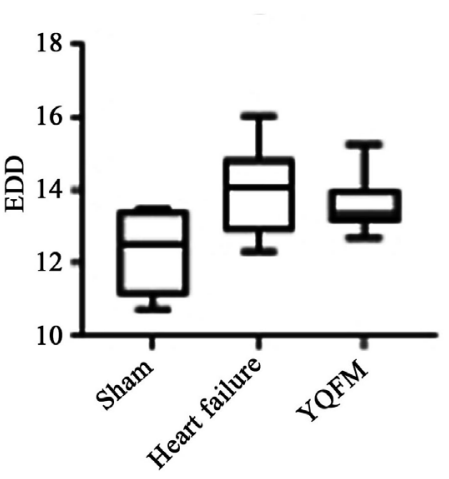

(a)

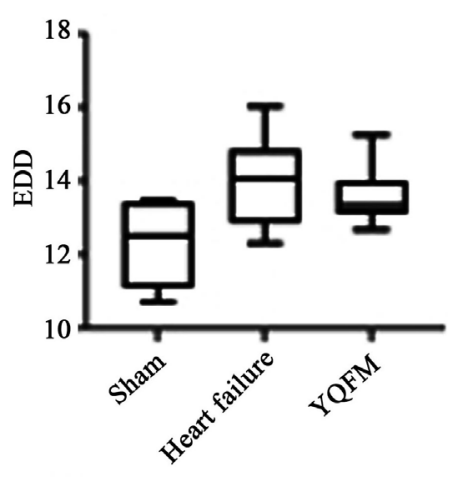

(c)

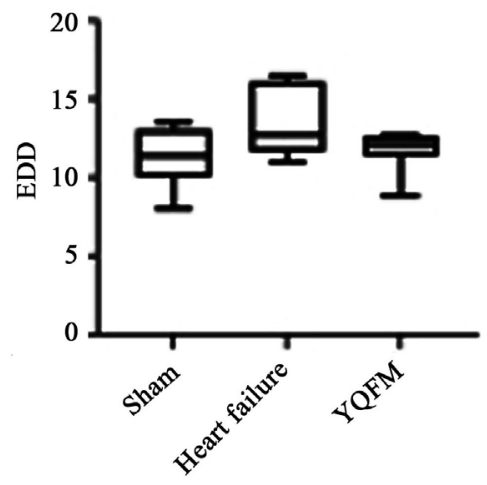

(e)

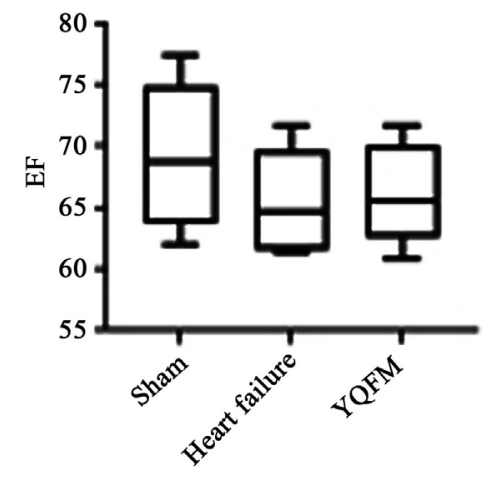

(b)

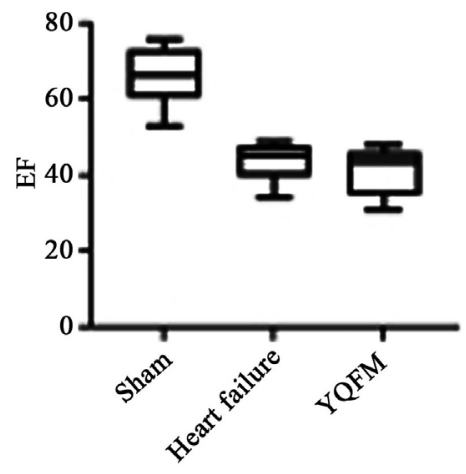

(d)

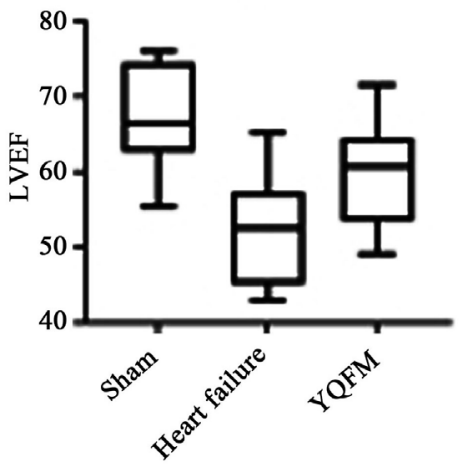

(f)

Figure 2. LVEF and LVEDD values preoperatively, and at 2 and 6 weeks after operation. (a) LVEDD preoperation (Sham group, Heart failure group, YQFM group); (b) LVEF preoperation (Sham group, Heart failure group, YQFM group); (c) LVEDD 2 weeks (Sham group, Heart failure group, YQFM group); (d) LVEF 2 weeks (Sham group, Heart failure group, YQFM group); (e) LVEDD 6 weeks (Sham group, Heart failure group, YQFM group); (f) LVEF 6 weeks (Sham group, Heart failure group, YQFM group).

At 6 weeks post-operation, the sham operation and heart failure groups showed no significant differences in BNP compared with values at 2 postoperative weeks, In the YiQiFuMai group, serum BNP at 6 postoperative weeks was significantly lower than that of the same group at 2 weeks post-operation. Compared with the heart failure group, there was no statistically significant difference in the YiQiFuMai groups in serum BNP levels (Figure 3). 


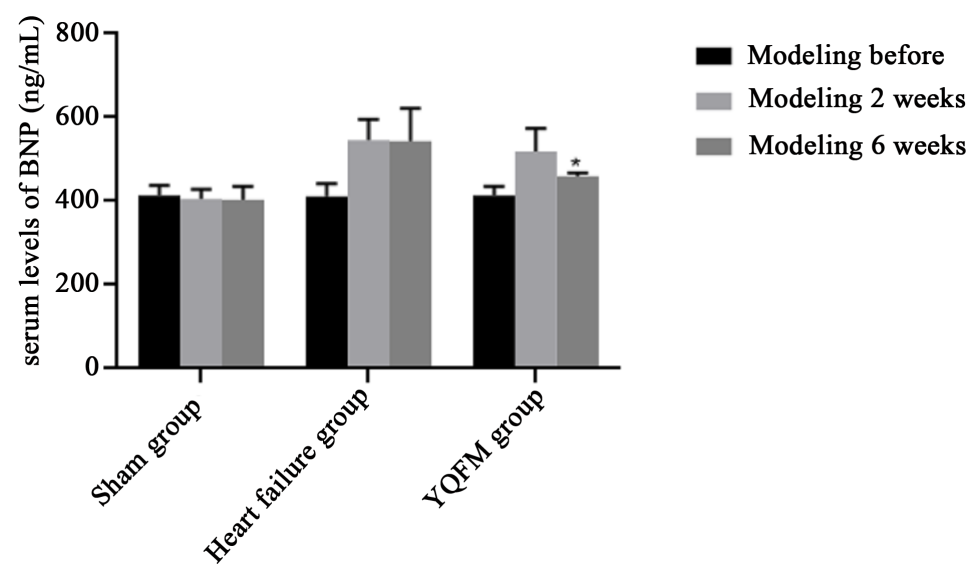

Figure 3. Serum BNP levels. Serum BNP was measured by ELISA in the sham, heart failure and YiQiFuMai groups preoperatively, and 2 and 6 weeks after the operation. ${ }^{\star} \mathrm{P}<$ 0.05 vs heart failure group at the same time point.

\section{Pathological damage in the myocardial tissue is reduced by YiQiFuMai}

In the heart failure group, $H \& E$ staining showed disordered arrangement of myocardial fibers, lysed cardiomyocyte nuclei, and red-stained cytoplasm. Myocardial edema, cell degeneration in the infarcted areas, and peripheral infiltration of inflammatory cells were also observed. Compared with the heart failure group, YiQiFuMai groups had reduced disease severity (Figure 4).

Treatment with YiQiFuMai increases capillary density after heart failure

Brown capillary endothelial cells stained by CD31 were visible in the myocardial tissues of each group six weeks post-operation. Compared with the heart failure group, theYiQiFuMai groups showed elevated capillary densities (Figure 5).

Improved ultrastructure and microcirculation of the myocardium by $Y$ iQiFuMai

Transmission electron microscopy showed that the sham-operated group had orderly arranged myofilaments, with abundant mitochondria arranged along myocardial fibers (Figure 6). Then, the myocardial microvascular ultrastructure was also assessed (Figure 7). The sham group had smooth myocardial microvascular lumen and intact basal lamina (Figure 7). In addition, there were pinocytotic vesicles in the cytoplasm and tight connections between endothelial cells in the sham group (Figure 7). In the heart failure group, the myocardial tissue ultrastructure showed broken myofilaments, disordered arrangement of the mitochondria, disappearing ridge, and vacuolar degeneration (Figure 6). For myocardial microvascular structure, the heart failure group had irregular microvascular lumen, broken basal lamina, and reduced number of pinocytotic vesicles (Figure 6). In the YiQiFuMai groups, the above abnormalities were improved (Figure 6 and Figure 7).

The mRNA expression levels of eNOS and VEGF are increased while ICAM-1 gene expression is decreased in infarcted areas of myocardial tissues from the YiQiFuMai groups 


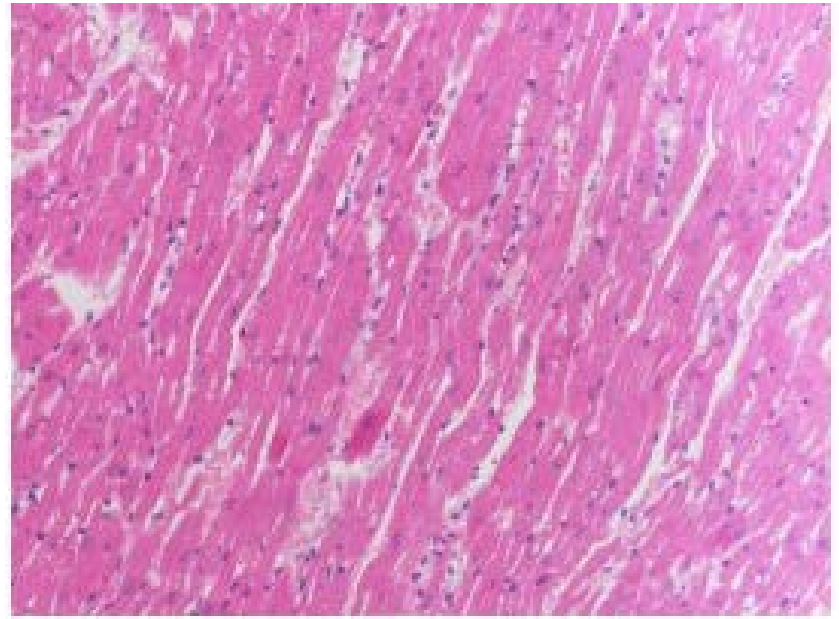

(a)

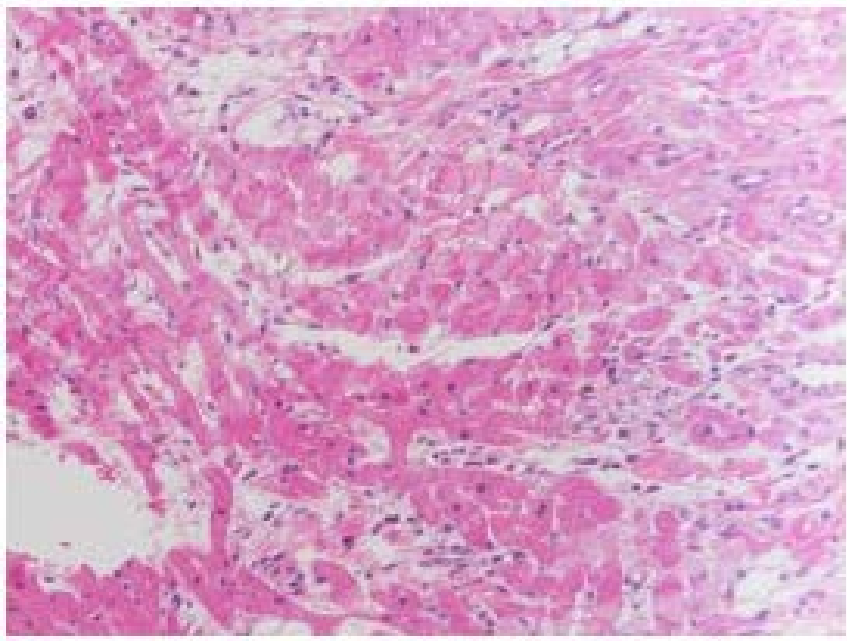

(b)

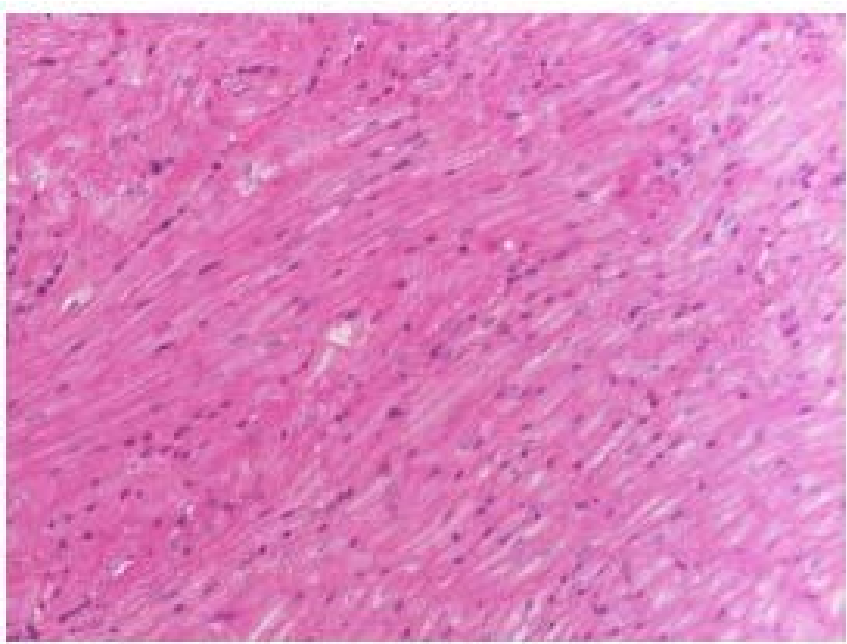

(c)

Figure 4. YiQiFuMai improve myocardial pathology. H\&E staining of myocardial tissue samples from the sham (a), heart failure $(b)$ and YiQiFuMai $(c)$ groups $(10 \times 20)$ six weeks after the operation. 


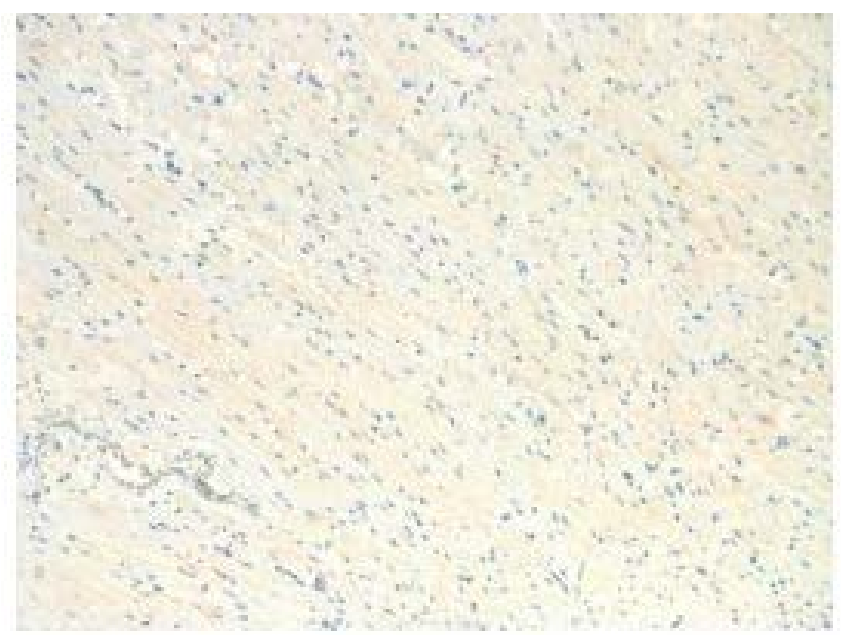

(a)

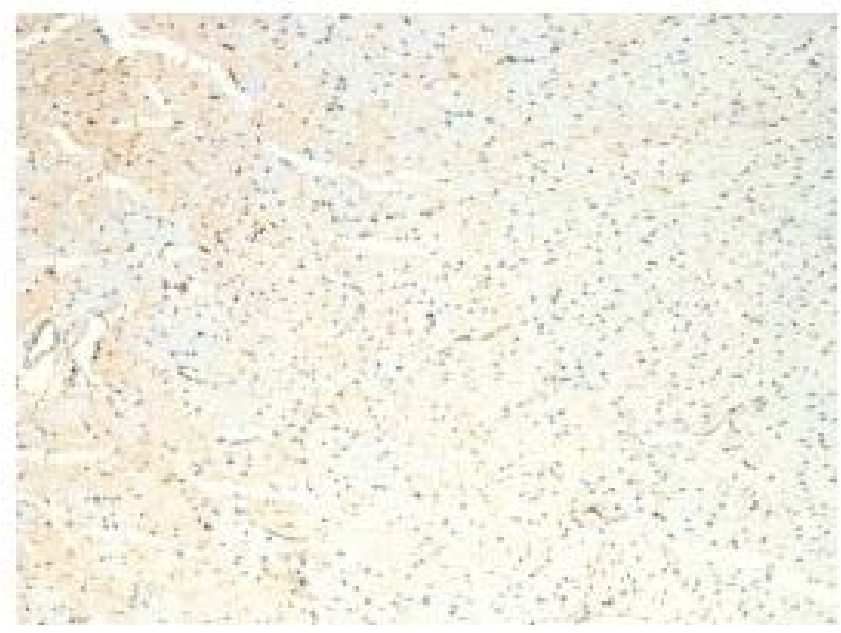

(b)

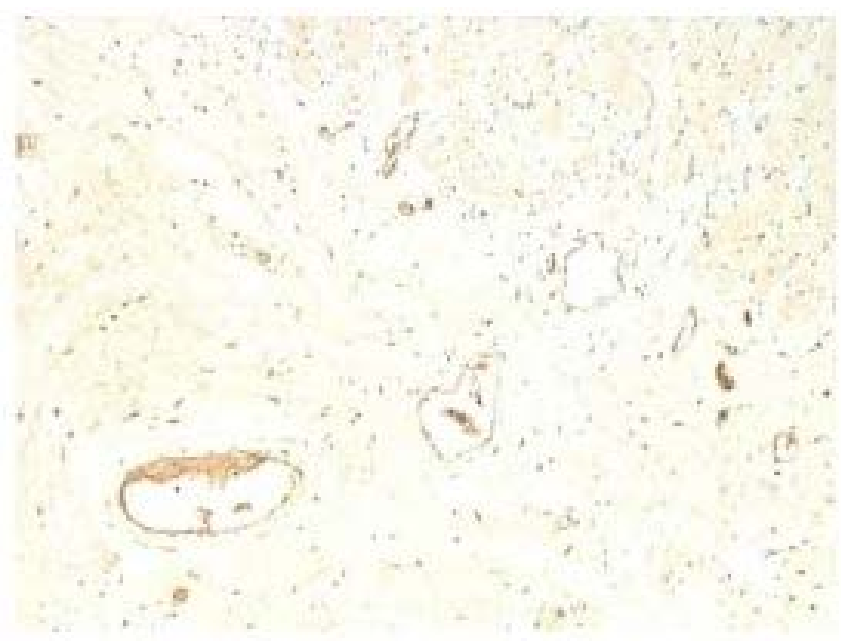

(c)

Figure 5. YiQiFuMai increase the amounts of capillary endothelial cells. Immunohistochemical staining of CD31 in myocardial tissue samples from the sham (a), heart failure (b) and YiQiFuMai $(c)$ groups $(10 \times 20)$ six weeks after the operation. 

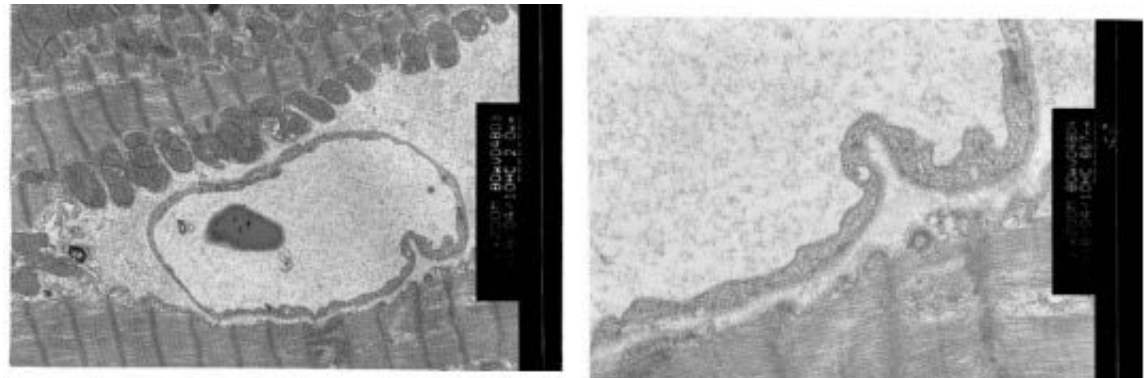

(a)
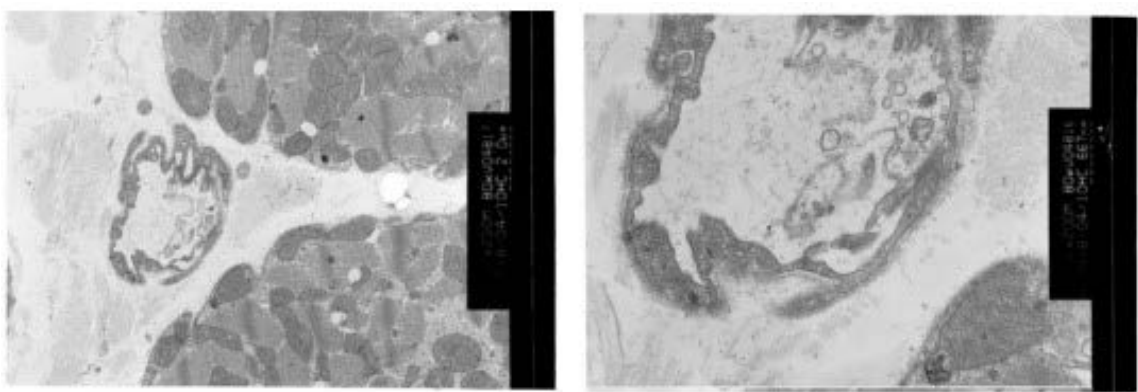

(b)
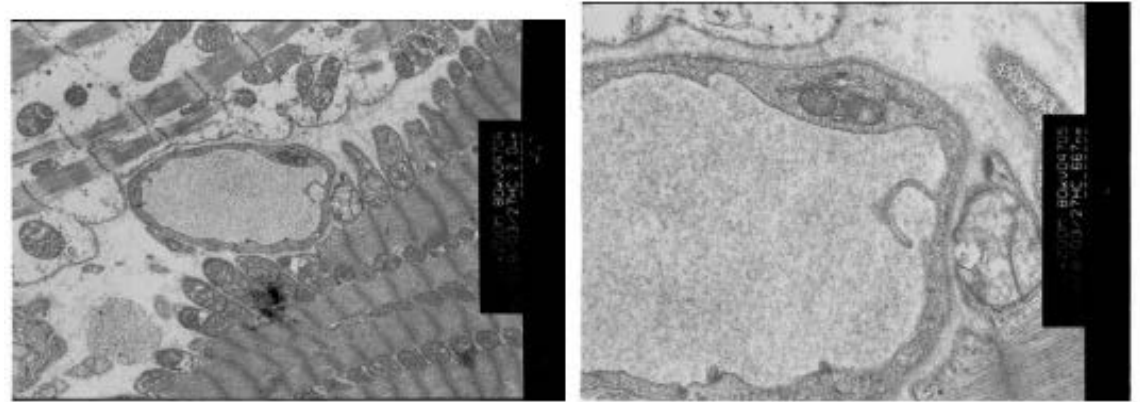

(c)

Figure 6. YiQiFuMai improve myocardial structure. Representative transmission electron micrographs of myocardial tissue samples from the sham (a), heart failure (b) and YiQiFuMai (c) groups six weeks after the operation (left: $5000 \times$, right: 15,000×).

To further investigate the molecular mechanisms underlying the observed phenotype, the mRNA expression levels of endothelial nitric oxide synthase (eNOS), ICAM-1, and VEGF were analyzed in infarcted areas of myocardial tissues. The heart failure group showed a trend towards decreased eNOS and increased ICAM-1 mRNA levels compared with the sham group (Figure 8). VEGF expression was similar between the two groups (Figure 8). Compared with the heart failure group, the YiQiFuMai group had significantly elevated eNOS levels (Figure 8). Furthermore, decreased ICAM-1 gene expression and increased VEGF mRNA amounts were detected in YiQiFuMai group compared with the heart failure group (Figure 8).

$A K T$ activation and eNOS and VEFG protein levels are increased, while ICAM-1 protein amounts are reduced in the infarcted areas of myocardial tissues from the YiQiFuMai group 

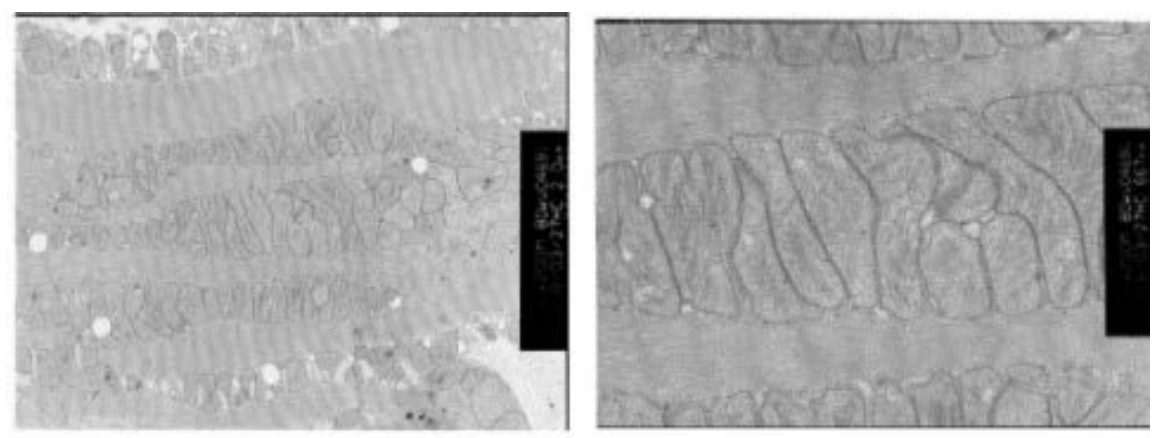

(a)
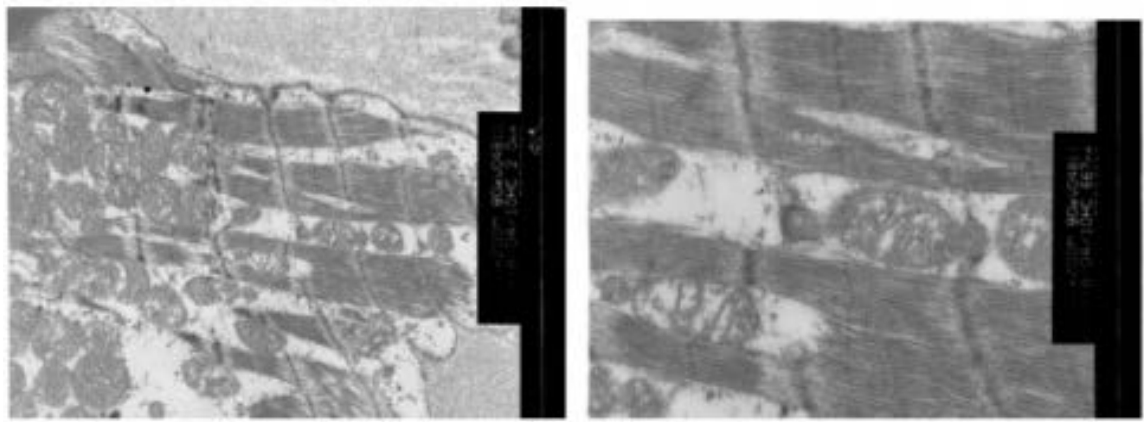

(b)
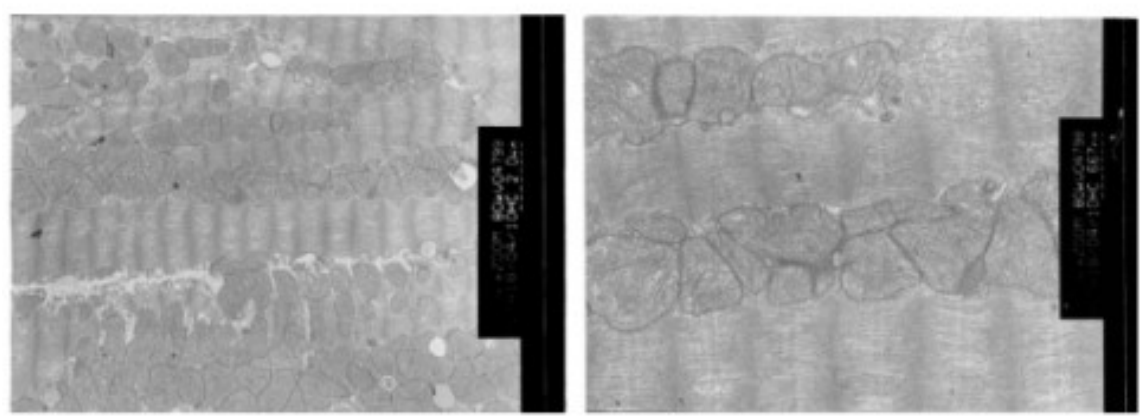

(c)

Figure 7. YiQiFuMai improve myocardial microvascular structure. Representative transmission electron micrographs of myocardial microvascular structure in the sham (a), heart failure (b) and YiQiFuMai (c) groups six weeks after the operation (left: 5000×, right: $15,000 \times)$.

Consistent with mRNA data, the heart failure group had reduced protein levels of eNOS, increased ICAM-1 protein amounts, and similar protein levels of VEGF compared with the sham group. eNOS is considered a downstream target of the PI3K/AKT signaling pathway. We found that $\mathrm{p}$-AKT protein levels were decreased in the heart failure group suggesting reduced activation of the AKT pathway after heart failure (Figure 9).

The YiQiFuMai group showed decreased ICAM-1 protein amounts and increased p-AKT and VEGF levels compared with the heart failure group. For eNOS protein levels, treatment with $t$ YiQiFuMai had no upregulation compared with the heart failure group (Figure 9). 

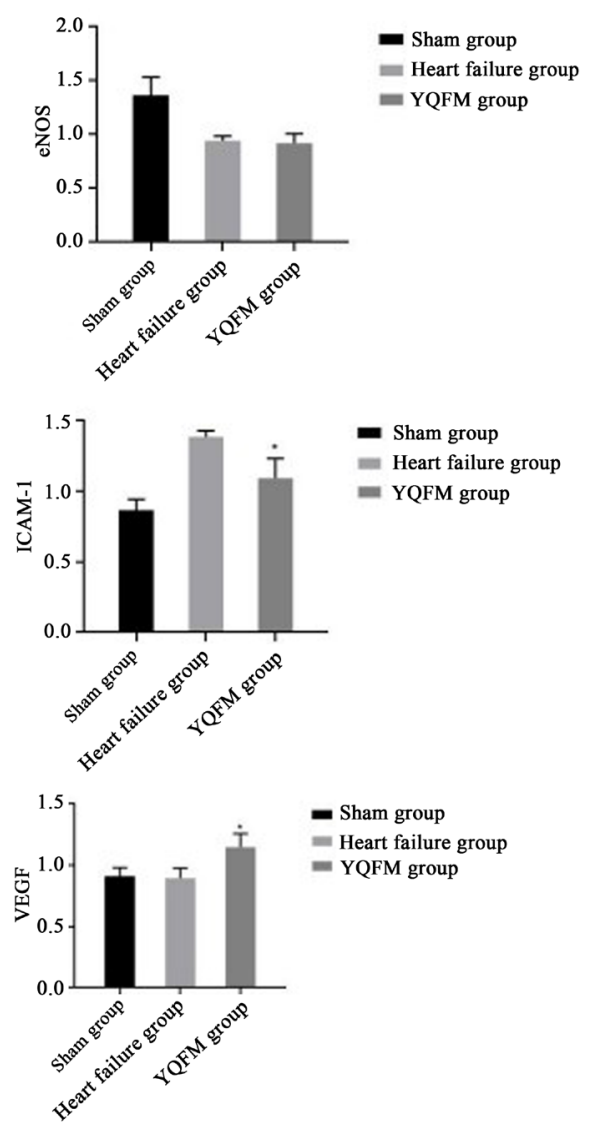

Figure 8. Increased eNOS and VEGF mRNA levels, and decreased ICAM-1 gene expression in response to YiQiFuMai treatments. Real-time qPCR analysis of genes encoding eNOS, ICAM- 1 and VEGF six weeks after the operation. ${ }^{\star} \mathrm{P}<0.05$ vs heart failure group.
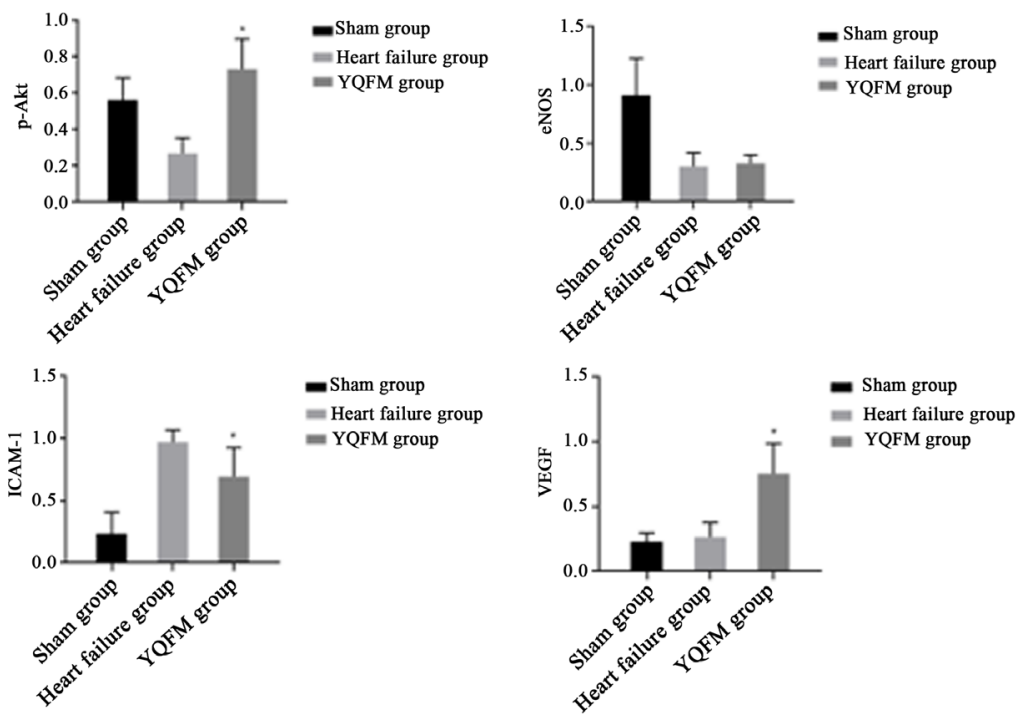

Figure 9. Increased AKT activation and VEGF protein levels, and decreased ICAM-1 protein amounts in response to YiQiFuMai treatments. Immunoblotting analysis of the pAKT, eNOS, ICAM-1, and VEGF proteins in the sham, heart failure, and YiQiFuMai groups 6 weeks after the operation. $\beta$-actin was used as a loading control. ${ }^{\star} \mathrm{P}<0.05$ vs heart failure group. 


\section{Discussion}

It was suggested that microvascular dysfunction is one of the major processes in the development of ischemic heart failure [12]. The endothelium of coronary vessels could regulate coronary blood flow in epicardial coronary and intramyocardial microcirculations [13]. Coronary blood flow increase partly depends on the regulation of endothelial function. It can be speculated that microvascular dysfunction may lead to recurrent episodes of myocardial ischemia and small infarctions that eventually result in heart failure.

Endothelial dysfunction occurs when myocardial NO generation decreases due to eNOS uncoupling and excessive peroxide production followed by nitrogen oxide activation [14]. Recent studies suggested that eNOS is a potential target of PI3K/AKT signaling. The activation of AKT leads to serine 1177 phosphorylation of eNOS, which can upregulate the expression of eNOS. In addition, activation of the PI3K/AKT/eNOS pathway can inhibit apoptosis in vascular endothelial cells [15]. AKT plays an important role in reducing cardiomyocyte apoptosis and myocardial protection. Activation of the PI3K/AKT pathway and upregulation of eNOS expression suppresses pro-apoptotic factors and activates the anti-apoptotic pathway to maintain the stability of the outer mitochondrial membrane and improve mitochondrial energy metabolism, thus inhibiting cell apoptosis and protecting the myocardium [16]. In this study, we showed that YiQiFuMai could provide protection to endothelial cells by reducing endothelial cell apoptosis and endothelial dysfunction. These events would eventually improve myocardial microcirculation and cardiac function.

Left ventricular dysfunction and heart failure are closely related to increased proinflammatory cytokine and ICAM-1 levels [17]. ICAM-1 is an immunoglobulin-like adhesion molecule, which is expressed during inflammatory stimulation of endothelial blood vessels. ICAM-1 induces inflammatory reactions by mediating white blood cell migration to the inflammatory site and adhesion to endothelial blood vessels [18]. Recent studies suggested that ICAM-1 expression in the heart and coronary endothelium can cause damage to the endothelium of coronary arteries, thus worsening the disorder of coronary microcirculation [19]. The present study showed that ICAM-1 expression was increased significantly in heart failure, and YiQiFuMai treatments downregulated ICAM-1 in vascular endothelial cells, which may reduce the damage to the vascular endothelium, thereby protecting cardiac microcirculation.

Angiogenesis is essential for myocardial cell survival and ventricular function recovery after myocardial infarction. A study by Chen et al. revealed that excessive expression of VEGF can inhibit cardiomyocyte apoptosis [20]. Ischemic reactions can induce the expression and activation of VEGF, causing microvascular dilation and neovascularization [21]. A study by Shen et al. showed that VEGF is excessively expressed in hypertrophic cardiomyocytes induced by pressure overload, which stimulates cardiac microvascular endothelial cells and induces neovascularization. This process may be mediated by activated 
PI3K/AKT/ Hif-1a pathway [22]. Zou et al. also demonstrated that upregulation of VEGF in the rat model of acute myocardial infarction stimulates ROS production, which in turn induces oxidative stress in the endoplasmic reticulum, as well as autophagy, thereby promoting cardiac neovascularization [23]. This study revealed that VEGF protein amounts were increased after treatment with YiQiFuMai. CD31 expression was also increased, suggesting that both treatments could potentially improve microcirculation by promoting neovascularization.

This study showed microvascular structure damage and dysfunction in ischemic heart failure. After application of the above drugs, the ejection fraction was improved, and BNP levels were decreased. Moreover, cardiac and microvascular structures were also improved as assessed by pathology and electron microscopy. Improving microcirculation to reverse cardiac dysfunction has been recently recognized as a promising clinical intervention in heart diseases. VEGF expression was also increased by YiQiFuMai. These events together are thought to protect microcirculation and improve ventricular remodeling. Further studies using PI3K or Akt inhibitors may directly reveal the relationship between eNOS and the PI3k/AKT pathway. In addition, the optimal drug concentrations required to treat the disease should be further investigated. Overall, this study observed microcirculatory alterations during the development of ischemic heart failure, which is associated with microvascular endothelial dysfunction. YiQiFuMai improved myocardial pathology and microcirculation, potentially via induced PI3K/AKT/eNOS pathway and VEGF upregulation.

\section{Conflicts of Interest}

The authors declare no conflicts of interest regarding the publication of this paper.

\section{References}

[1] Redfield, M.M. (2017) Heart Failure with Preserved Ejection Fraction. The New England Journal of Medicine, 376, 897. https://doi.org/10.1056/NEJMc1615918

[2] Metra, M. and Teerlink, J.R. (2017) Heart Failure. The Lancet, 390, 1981-1995. https://doi.org/10.1016/S0140-6736(17)31071-1

[3] Sestito, A., et al. (2011) Relation between Cardiovascular Risk Factors and Coronary Microvascular Dysfunction in Cardiac Syndrome X. Journal of Cardiovascular Medicine, 12, 322-327. https://doi.org/10.2459/JCM.0b013e3283406479

[4] Fleming, I. (2010) Molecular Mechanisms Underlying the Activation of eNOS. Pflügers Archiv, 459, 793-806. https://doi.org/10.1007/s00424-009-0767-7

[5] Engin, A. (2017) Endothelial Dysfunction in Obesity. Advances in Experimental Medicine and Biology, 960, 345-379. https://doi.org/10.1007/978-3-319-48382-5 15

[6] Yoo, S.Y. and Kwon, S.M. (2013) Angiogenesis and Its Therapeutic Opportunities. Mediators of Inflammation, 2013, Article ID: 127170. https://doi.org/10.1155/2013/127170

[7] Koch, S., et al. (2011) Signal Transduction by Vascular Endothelial Growth Factor Receptors. Biochemical Journal, 437, 169-183. https://doi.org/10.1042/BJ20110301 
[8] Greenberg, D.A. and Jin, K. (2013) Vascular Endothelial Growth Factors (VEGFs) and Stroke. Cellular and Molecular Life Sciences, 70, 1753-1761. https://doi.org/10.1007/s00018-013-1282-8

[9] Li, F., Tan, Y.S., Chen, H.L., Yan, Y., Zhai, K.F., Li, D.P., et al. (2015) Identification of Schisandrin as a Vascular Endothelium Protective Component in YiQiFuMai Powder Injection Using HUVECs Binding and HPLC-DAD-Q-TOF-MS/MS Analysis. Journal of Pharmacological Sciences, 129, 1-8. https://doi.org/10.1016/j.jphs.2015.02.003

[10] Zhang, Y., et al. (2019) YiQiFuMai Powder Injection Ameliorates Chronic Heart Failure through Cross-Talk Between Adipose Tissue and Cardiomyocytes via up-Regulation of Circulating Adipokine Omentin. Biomedicine \& Pharmacotherapy, 119, Article ID: 109418. https://doi.org/10.1016/j.biopha.2019.109418

[11] Zhang, Y., et al. (2019) YiQiFuMai Powder Injection Attenuates Coronary Artery Ligation-Induced Heart Failure Through Improving Mitochondrial Function via Regulating ROS Generation and CaMKII Signaling Pathways. Frontiers in Pharmacology, 10, 381. https://doi.org/10.3389/fphar.2019.00381

[12] Li, S., et al. (2019) Perindopril and a Galectin-3 Inhibitor Improve Ischemic Heart Failure in Rabbits by Reducing Gal-3 Expression and Myocardial Fibrosis. Frontiers in Physiology, 10, 267. https://doi.org/10.3389/fphys.2019.00267

[13] den Uil, C.A., et al. (2008) The Microcirculation in Health and Critical Disease. Progress in Cardiovascular Diseases, 51, 161-170.

https://doi.org/10.1016/j.pcad.2008.07.002

[14] Malakul, W., et al. (2018) Naringin Ameliorates Endothelial Dysfunction in Fructose-Fed Rats. Experimental and Therapeutic Medicine, 15, 3140-3146. https://doi.org/10.3892/etm.2018.5759

[15] Arab, H.H., Salama, S.A. and Maghrabi, I.A. (2018) Camel Milk Ameliorates 5-Fluorouracil-Induced Renal Injury in Rats: Targeting MAPKs, NF-kappaB and PI3K/Akt/eNOS Pathways. Cellular Physiology and Biochemistry, 46, 1628-1642. https://doi.org/10.1159/000489210

[16] Li, J.B., et al. (2017) Overexpression of microRNA-138 Alleviates Human Coronary Artery Endothelial Cell Injury and Inflammatory Response by Inhibiting the PI3K/Akt/eNOS Pathway. Journal of Cellular and Molecular Medicine, 21, 1482-1491. https://doi.org/10.1111/jcmm.13074

[17] Salvador, A.M., et al. (2016) Intercellular Adhesion Molecule 1 Regulates Left Ventricular Leukocyte Infiltration, Cardiac Remodeling, and Function in Pressure Overload-Induced Heart Failure. Journal of the American Heart Association, 5, e003126. https://doi.org/10.1161/JAHA.115.003126

[18] Pasqui, A.L., et al. (2005) Structural and Functional Abnormality of Systemic Microvessels in Cardiac Syndrome X. Nutrition, Metabolism \& Cardiovascular Diseases, 15, 56-64. https://doi.org/10.1016/j.numecd.2004.05.001

[19] Caprio, M., et al. (2008) Functional Mineralocorticoid Receptors in Human Vascular Endothelial Cells Regulate Intercellular Adhesion Molecule-1 Expression and Promote Leukocyte Adhesion. Circulation Research, 102, 1359-1367. https://doi.org/10.1161/CIRCRESAHA.108.174235

[20] Chen, X.G., et al. (2016) Vascular Endothelial Growth Factor-C Protects Heart from Ischemia/Reperfusion Injury by Inhibiting Cardiomyocyte Apoptosis. Molecular and Cellular Biochemistry, 413, 9-23. https://doi.org/10.1007/s11010-015-2622-9

[21] Lahteenvuo, J.E., et al. (2009) Vascular Endothelial Growth Factor-B Induces Myo- 
cardium-Specific Angiogenesis and Arteriogenesis via Vascular Endothelial Growth Factor Receptor-1- and Neuropilin Receptor-1-Dependent Mechanisms. Circulation, 119, 845-856. https://doi.org/10.1161/CIRCULATIONAHA.108.816454

[22] Shen, J., et al. (2018) Increased Myocardial Stiffness Activates Cardiac Microvascular Endothelial Cell via VEGf Paracrine Signaling in Cardiac Hypertrophy. Journal of Molecular and Cellular Cardiology, 122, 140-151.

[23] Zou, J., et al. (2019) VEGF-A Promotes Angiogenesis after Acute Myocardial Infarction through Increasing ROS Production and Enhancing ER Stress-Mediated Autophagy. Journal of Cellular Physiology, 234, 17690-17703. 